\title{
Electromicrobiology: Electron Transfer via Biowires in Nature and Practical Applications
}

\author{
Derek Lovley $^{\text {a }}$ \\ ${ }^{1}$ Department of Microbiology, University of Massachusetts, Amherst, MA 01003-9298, USA
}

\begin{abstract}
One of the most exciting developments in the field of electromicrobiology has been the discovery of electrically conductive pili (e-pili) in Geobacter species that transport electrons with a metallic-like mechanism. The e-pili are essential for extracellular electron transport to Fe(III) oxides and longrange electron transport through the conductive biofilms that form on the anodes of microbial fuel cells. The e-pili also facilitate direct interspecies electron transfer between Geobacter and Methanosaeta or Methanosarcina species. Metatranscriptomic studies have demonstrated that Geobacter/Methanosaeta DIET is an important process in anaerobic digesters converting brewery wastes to methane. Increasing e-pili expression through genetic modification of regulatory systems or adaptive evolution yields strains with enhanced rates of extracellular electron transfer. Measurement of the conductivity of individual e-pili has demonstrated that they have conductivities higher than those of a number of synthetic conducting organic polymers. Multiple lines of evidence have demonstrated that aromatic amino acids play an important role in the electron transport along e-pili, suggesting opportunities to tune e-pili conductivity via genetic manipulation of the amino acid composition of e-pili. It is expected that e-pili will be harnessed to improve microbe-electrode processes such as microbial electrosynthesis and for the development of novel biosensors. Also, e-pili show promise as a sustainable 'green' replacement for electronic materials that contain toxic components and/or are produced with harsh chemicals.
\end{abstract}

\footnotetext{
${ }^{\mathrm{a}}$ Corresponding author: $\underline{\text { dlovley@microbio.umass.edu }}$
} 